\title{
Relationship Between Q-Angle and Articular Cartilage in Female Patients With Symptomatic Knee Osteoarthritis: Ultrasonographic and Radiologic Evaluation
}

\author{
Ayşe Aydemir EKİM, ${ }^{1}$ Hatice HAMARAT, ${ }^{2}$ Ahmet MUSMUL ${ }^{3}$ \\ ${ }^{1}$ Department of Physical Medicine and Rehabilitation, Eskişehir State Hospital, Zübeyde Hanım Campus, Eskişehir, Turkey, \\ ${ }^{2}$ Department of Internal Medicine, Eskişehir State Hospital, Eskişehir, Turkey \\ ${ }^{3}$ Department of Biostatistics and Medical Informatics, Medical Faculty of Osmangazi University, Eskişehir, Turkey
}

\begin{abstract}
Objectives: This study aims to examine the association between Q-angle and clinical, radiological, and ultrasonographic findings in patients with knee osteoarthritis (OA).

Patients and methods: Sixty-eight female patients (mean age $59.8 \pm 6.8$ years; range 39 to 78 years) diagnosed with knee OA were included in this study and classified into two groups according to Q-angle of symptomatic knees: low Q-angle group (LQ) ( $\mathrm{n}=40$ ) and high $\mathrm{Q}$-angle group (HQ) ( $n=28$ ) (LQ-angle $<15^{\circ}$ and $\mathrm{HQ}$-angle $\geq 15^{\circ}$, respectively). Patients were clinically assessed for pain and functional status by using a visual analog scale and the Western Ontario and McMaster Universities Arthritis Index. X-rays of knees were scored using the Kellgren-Lawrence OA grading system. Symptomatic knees were also evaluated using ultrasonography for distal femoral cartilage thickness/grading.

Results: No significant difference was observed in clinical and imaging findings between the groups ( $p>0.05$ ). HQ-angle measurements were positively correlated with cartilage grading by ultrasonography $(r=0.435, p=0.033)$ and Kellgren-Lawrence grading system ( $r=0.435$, $p=0.021)$, and negatively correlated with cartilage thickness measurements of the medial femoral condyle $(r=-0.399, p=0.036)$.

Conclusion: We found that HQ-angle was associated with cartilage thickness measurements of the medial femoral condyle and cartilage grading by ultrasonography and the Kellgren-Lawrence grading system in patients with knee OA.

Keywords: Articular; cartilage; osteoarthritis of knee; ultrasound imaging.
\end{abstract}

The quadriceps angle, or Q-angle, is defined as the angle formed by the intersection of two lines, one that starts at the anterior iliac spine and goes to the center of the patella, and another that goes from the tibial tuberosity to the center of the patella. ${ }^{1}$ When compared to individuals with low $\mathrm{Q}$-angle (LQ-angle $\left.<15^{\circ}\right)$, asymptomatic individuals with high Q-angle ( $\mathrm{HQ}$-angle $\geq 15^{\circ}$ ) displayed anatomical cross-sectional areas of the vastus medialis and lateralis that were approximately $10 \%$ smaller when using the $\mathrm{Q}$-angle as an estimate of quadriceps muscle disorientation..$^{2,3}$ As a consequence, contrary to common belief, 4,5 the force exerted by an almost equally atrophied vastus lateralis may successfully counteract the lower force exerted on the patella by an atrophied vastus medialis. Lateral maltracking of the patella and possible wear of the neighboring articular cartilage may thus be prevented.

Osteoarthritis (OA) is the most common type of joint disorder affecting the majority of people and it causes a huge burden of pain and disability. ${ }^{6}$ The most common properties of $\mathrm{OA}$ are loss of cartilage structure, subchondral bone sclerosis, synovial inflammation, and osteophyte formation, with involvement of the whole joint (i.e. joint failure). ${ }^{7}$ Some authors have found 
that, in comparison to the lateral tibiofemoral cartilage, the thickness of the medial tibiofemoral and patellofemoral articular cartilages in healthy individuals with normally aligned knees is thinner. ${ }^{8,9}$ This is likely due to differences in the distribution of loads over the medial and lateral compartments. ${ }^{10,11}$ Although an altered load distribution between the lateral and medial compartments of the joint may be demonstrated in individuals with varus or valgus knees (and an inevitably $\mathrm{HQ}$-angle), it remains unknown whether the thickness of the articular cartilages in these individuals is also functionally adapted, particularly at an early age. Clinicians have taken advantage of this information to develop intervention strategies to prevent progressive wear of the knee cartilages. ${ }^{10,11}$

The imaging modalities most frequently used in this field are conventional radiography, and within the last decade, ultrasonography (US). ${ }^{12}$ Because of its convenience and low expense, in addition to providing dynamic assessment and guidance during interventions and the absence of radiation, musculoskeletal US has already taken its place in the daily practice of physical and rehabilitation physicians. ${ }^{13}$

Taking into consideration all the information above, in this study, we aimed to examine the association between Q-angle and clinical, radiological, and ultrasonographic findings in patients with knee OA.

\section{PATIENTS AND METHODS}

A total of 68 female patients (mean age $59.8 \pm 6.8$ years; range 39 to 78 years) were recruited from Eskişehir State Hospital, Zübeyde Hanım Campus Physical Medicine and Rehabilitation outpatient clinic between January 2016 and March 2016. All patients with a diagnosis of knee OA according to criteria established by the American College of Rheumatology were enrolled. ${ }^{14}$ The study protocol was approved by the Medical Faculty of Eskişehir Osmangazi University Ethics Committee. A written informed consent was obtained from all participants. The study was conducted in accordance with the principles of the Declaration of Helsinki.

Subjects with postural deviations such as leg length discrepancy, recurvatum knees, muscle shortening, kyphosis and/or scoliosis, past surgery, injury of the lower limbs, patellofemoral pain syndrome, thyroid dysfunction, rheumatic conditions, obesity with body mass index $>30 \mathrm{~kg} / \mathrm{m}^{2}$, increased level of kinetic activity or who used anabolic drugs and/or participated in organized athletic activities were excluded from this study.

Patients were divided into a LQ-angle group $(n=40)$ and an HQ-angle group $(n=28)$ based on the magnitude of $\mathrm{Q}$-angle in the symptomatic limb (LQ-angle $<15^{\circ}$ and $\mathrm{HQ}$-angle $\geq 15^{\circ}$, respectively).

All patients were evaluated in terms of age, height, weight, and duration of symptoms by a research physician. Laboratory testing comprised erythrocyte sedimentation rate and serum C-reactive protein. None of the patients had redness, swelling, or instability of joints upon physical examination and all of them were postmenopausal. Each patient was clinically assessed for pain and functional status using a visual analog scale at rest and at motion and the Western Ontario and McMaster Universities Arthritis Index, which is a three-dimensional, disease-specific, and self-administered health status measure that evaluates pain, joint stiffness, and physical function in patients with knee OA. In this study, the Turkish version of Western Ontario and McMaster Universities Arthritis Index was used. ${ }^{15}$

The $\mathrm{Q}$-angle was measured in the symptomatic lower leg of the subjects using a commercially available full circle goniometer. Each subject was instructed to lie in supine position with the knees fully extended and the quadriceps relaxed. As the magnitude of the $\mathrm{Q}$-angle may be influenced by the position of the foot in terms of inward-outward rotation, ${ }^{16}$ the foot was positioned such that the line connecting the middle of the heel with the second metatarsal was perpendicular to the ground. ${ }^{17}$ Three landmarks ( $8 \mathrm{~mm}$ diameter) were located by palpation: the anterior superior iliac spine, the center of the patella and the tibial tubercle. The patellar center was located at the intersection of a mediolateral line extending through the widest part of the patella and a superoinferior line connecting the base and the tip of the patella. The long arm of the goniometer was placed along the line connecting the anterior superior 
iliac spine with the center of the patella and the short arm along the line connecting the center of the patella with the tibial tubercle.

All measurements were performed by the same physiatrist using a $5-10 \mathrm{MHz}$ linear probe (Diagnostic Ultrasound System, Shimadzu, Kyoto, Japan). All knees were evaluated for distal femoral cartilage thickness/grading. Distal femoral cartilage assessment was performed while patients were lying in a supine position with their knees in maximum flexion. The transducer was placed axially above the patellar outer edge.18 Cartilage thickness measurements were taken from the mid-points of the medial femoral condyle, intercondylar area, and the lateral femoral condyle. The cartilage thickness was measured as the distance between the thin hyperechoic line at the synovial space-cartilage interface and the sharp hyperechoic line at the cartilage-bone interface. ${ }^{19}$ Cartilage grading (range 0-6) was conducted by evaluation of sharpness, clarity, and thickness of the cartilage band. ${ }^{18}$

Knee radiographs were evaluated using the Kellgren-Lawrence (K-L) grading system, the most widely used method for the diagnosis of knee OA, focusing on osteophytes and/or joint space narrowing. ${ }^{20}$

\section{Statistical analysis}

All data analyses were performed using IBM SPSS version 21.0 software (IBM Corp., Armonk, NY, USA). Data were expressed as the mean \pm standard deviation for nominal variables and as median (25-75\%) for ordinal variables. The normal distribution and homogeneity of each parameter was tested with independent samples t-tests. The Mann-Whitney U test was used for data with non-normal distributions. Categorical variables (i.e., K-L grading) were evaluated with the Chi-square test. Since the parameters were not normally distributed according to the ShapiroWilk test, the Spearman's correlation was used. Correlations among Q-angle and clinical and imaging findings were assessed by using the Spearman's correlation coefficient. Statistical significance was set at $p<0.05$.

\section{RESULTS}

No difference was observed in any of the parameters between the groups ( $\mathrm{p}>0.05)$ (Table 1).
Although there was no correlation between clinical characteristics, imaging findings and Q-angles of all participants, we found correlations between the $\mathrm{Q}$-angle measurements and cartilage grading by US ( $\mathrm{r}=0.435, \mathrm{p}=0.033), \mathrm{K}-\mathrm{L}$ grading

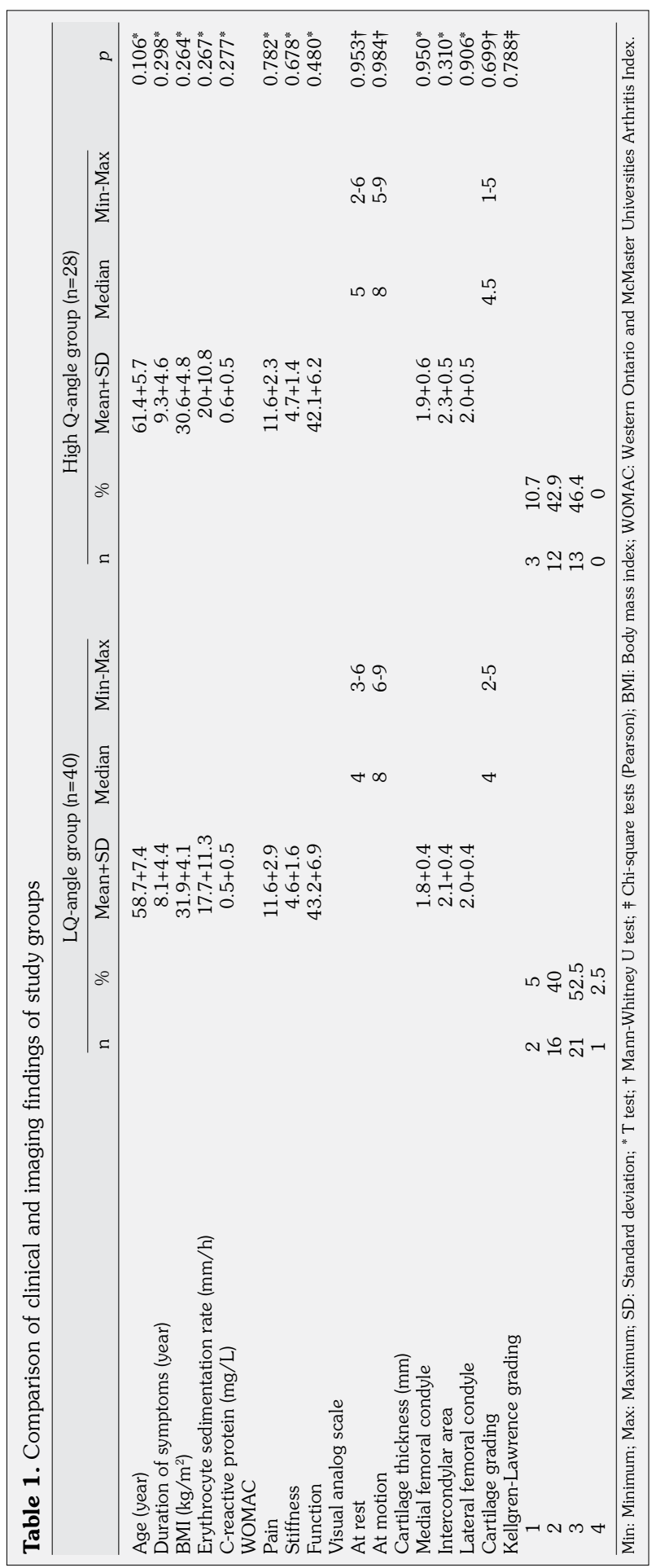


$(\mathrm{r}=0.435, \mathrm{p}=0.021)$ and medial femoral condyle thickness $(r=-0.399, p=0.036)$. Measurements of the 68 female patients' symptomatic knees were analyzed. Patients were divided into two group based on $\mathrm{Q}$ angle. The demographic characteristics, clinical and imaging findings of the patients are presented in Table 1 .

\section{DISCUSSION}

In this study, we evaluated the association between the Q-angle and clinical features and radiologic/ultrasonographic findings of patients with knee OA. According to our results, HQ-angle measurements were positively correlated with cartilage grading by US and K-L grading and were negatively correlated with medial femoral condyle.

The evidence that an increased (or decreased) Q-angle actually has an effect on the position of the patella and/or the thickness of the knee cartilages is limited. Mizuno et al. ${ }^{21}$ have shown in a biomechanical study on cadaveric knees that an increased Q-angle may shift the patella laterally when the knee is between $20^{\circ}$ and $60^{\circ}$ of flexion, leading to lateral patellar dislocation or increased lateral patellofemoral contact pressures. Biedert and Warnke, ${ }^{22}$ who also measured the position of the patella with computed tomography in patients with patellofemoral pain syndrome, failed to show a significant relationship between the $\mathrm{Q}$-angle and patellar positioning within the femoral trochlea.

Tsakoniti et al. ${ }^{23}$ revealed that healthy individuals with $\mathrm{HQ}$-angle are unlikely to demonstrate any changes in the position of the patella or the thickness of the knee articular cartilages. Patellar positioning and the thickness of the articular cartilages were determined in 19 asymptomatic male individuals with $\mathrm{HQ}$-angle using magnetic resonance imaging. The findings of their study rejected the hypothesis that the articular cartilages of the medial and lateral compartments of the knee may respond to an increased $\mathrm{Q}$-angle by adapting their thicknesses. Adaptive changes in the thickness of the articular cartilages of the knee may not have been detected in the $\mathrm{HQ}$-angle group due to the participants' age (mean age $=20.1$ years). ${ }^{23}$ It has been shown that age is negatively related to articular cartilage thickness (e.g. femoral condyles), especially in areas that are subject to compression due to body weight. ${ }^{24}$ This hypothesis was based on the fact that common malalignments of the lower limbs, such as valgus knees, a deformity that results in an increased $\mathrm{Q}$-angle, alter the pressure distribution over the medial and lateral compartments of the joint, ${ }^{10,11}$ a biomechanical factor that possibly contributes to articular cartilage wear and the initiation of knee OA. ${ }^{10,11,25}$ Bruns et al. ${ }^{10}$ in a cadaveric study reported that knee joint pressure distribution was shifted towards the lateral compartment with the joint positioned at $10^{\circ}$ valgus. Huberti and Hayes $^{25}$ in a similar study stressed that in knees with $\mathrm{Q}$-angles of $10^{\circ}$ more than normal (which is approximately $15^{\circ}$ ), the patellofemoral contact pressures were increased over the lateral facet of the patella in half of the knees under investigation.

In the present study, the medial femoral cartilage was thinner when compared to the lateral femoral cartilage. Likewise, our findings are in agreement with previous observations that have shown a decreased volume of the medial compartment of the knee when compared to the lateral compartment in patients with knee OA. ${ }^{8,9}$ It has been hypothesized that the thinner medial tibiofemoral and patellofemoral articular cartilages may actually demonstrate an adaptive response to the higher pressure that is applied on this area during normal everyday weight-bearing activities, such as standing ${ }^{26}$ and walking. ${ }^{11,27}$ This response may in part be explained by the mechanism that allows hyaline cartilage to expel water when subjected to high osmotic or hydrostatic pressure and conversely to ingress water from the synovial fluid when this pressure is low. ${ }^{26}$

The Q-angle is widely used as an indicator of patellofemoral problems such as patellofemoral pain syndrome. ${ }^{28}$ In patients with advanced OA of the knee, patellofemoral degeneration at the joint frequently coexists with tibiofemoral OA. Recent studies have shown that patients with concomitant patellofemoral OA had more symptoms and functional disabilities than those with isolated tibiofemoral OA. ${ }^{29,30}$

Musculoskeletal US is a non-invasive, widely available, and relatively inexpensive technique. It can be promptly performed and is easily accepted by patients, it is a radiation-free diagnostic test, and it does not have any contraindications. ${ }^{31}$ In a previous study, a good level of agreement in cartilage thickness measurements between 
magnetic resonance imaging and US was found. ${ }^{32,33}$ In our study, cartilage was graded and the distal femoral cartilage thickness of the symptomatic knee was measured in females with knee OA using US, and we found that HQ-angle measurements were correlated with cartilage grading/thickness by US.

The limitations of this study are the small number of patients included and its cross-sectional design.

In conclusion, this study shows that the relationship between knee cartilage thickness and $\mathrm{HQ}$-angle can be evaluated by US without using a radiographic method. However, further research is needed to investigate other factors affecting cartilage thickness and grade and Q-angle in knee OA.

\section{Declaration of conflicting interests}

The authors declared no conflicts of interest with respect to the authorship and/or publication of this article.

\section{Funding}

The authors received no financial support for the research and/or authorship of this article.

\section{REFERENCES}

1. Heiderscheit BC, Hamill J, Van Emmerik RE. Q-angle influences on the variability of lower extremity coordination during running. Med Sci Sports Exerc 1999;31:1313-9.

2. Hungerford DS, Barry M. Biomechanics of the patellofemoral joint. Clin Orthop Relat Res 1979;144:9-15.

3. Tsakoniti AE, Stoupis CA, Athanasopoulos SI. Quadriceps cross-sectional area changes in young healthy men with different magnitude of $\mathrm{Q}$ angle. $\mathrm{J}$ Appl Physiol (1985) 2008;105:800-4.

4. Gilleard W, McConnell J, Parsons D. The effect of patellar taping on the onset of vastus medialis obliquus and vastus lateralis muscle activity in persons with patellofemoral pain. Phys Ther 1998;78:25-32.

5. Grelsamer RP, Klein JR. The biomechanics of the patellofemoral joint. J Orthop Sports Phys Ther 1998;28:286-98.

6. Woolf AD, Pfleger B. Burden of major musculoskeletal conditions. Bull World Health Organ 2003;81:646-56.

7. Hunter DJ, Felson DT. Osteoarthritis. BMJ 2006;332:639-42.

8. Eckstein F, Winzheimer M, Hohe J, Englmeier KH, Reiser M. Interindividual variability and correlation among morphological parameters of knee joint cartilage plates: analysis with three-dimensional MR imaging. Osteoarthritis Cartilage 2001;9:101-11.

9. Jones G, Glisson M, Hynes K, Cicuttini F. Sex and site differences in cartilage development: a possible explanation for variations in knee osteoarthritis in later life. Arthritis Rheum 2000;43:2543-9.

10. Bruns J, Volkmer M, Luessenhop S. Pressure distribution at the knee joint. Influence of varus and valgus deviation without and with ligament dissection. Arch Orthop Trauma Surg 1993;113:12-9.

11. Harrington IJ. Static and dynamic loading patterns in knee joints with deformities. J Bone Joint Surg [Am] 1983;65:247-59.

12. Babyn PS. Pediatric musculoskeletal imaging. Semin Musculoskelet Radiol 2009;13:157.

13. Ozçakar L, Carli AB, Tok F, Tekin L, Akkaya N, Kara M. The utility of musculoskeletal ultrasound in rehabilitation settings. Am $J$ Phys Med Rehabil 2013;92:805-17.

14. Altman R, Asch E, Bloch D, Bole G, Borenstein $\mathrm{D}$, Brandt $\mathrm{K}$, et al. Development of criteria for the classification and reporting of osteoarthritis. Classification of osteoarthritis of the knee. Diagnostic and Therapeutic Criteria Committee of the American Rheumatism Association. Arthritis Rheum 1986;29:1039-49.

15. Basaran S, Guzel R, Seydaoglu G, Guler-Uysal F. Validity, reliability, and comparison of the WOMAC osteoarthritis index and Lequesne algofunctional index in Turkish patients with hip or knee osteoarthritis. Clin Rheumatol 2010;29:749-56.

16. Olerud $\mathrm{C}$, Berg P. The variation of the $\mathrm{Q}$ angle with different positions of the foot. Clin Orthop Relat Res 1984;191:162-5.

17. Guerra JP, Arnold MJ, Gajdosik RL. Q angle: effects of isometric quadriceps contraction and body position. J Orthop Sports Phys Ther 1994;19:200-4.

18. Lee CL, Huang MH, Chai CY, Chen CH, Su JY, Tien YC. The validity of in vivo ultrasonographic grading of osteoarthritic femoral condylar cartilage: a comparison with in vitro ultrasonographic and histologic gradings. Osteoarthritis Cartilage 2008;16:352-8.

19. Yoon $\mathrm{CH}$, Kim HS, Ju JH, Jee WH, Park SH, Kim HY. Validity of the sonographic longitudinal sagittal image for assessment of the cartilage thickness in the knee osteoarthritis. Clin Rheumatol 2008;27:1507-16.

20. Kellgren JH, Lawrence JS. Radiological assessment of osteo-arthrosis. Ann Rheum Dis 1957;16:494-502.

21. Mizuno Y, Kumagai M, Mattessich SM, Elias JJ, Ramrattan N, Cosgarea AJ, et al. Q-angle influences tibiofemoral and patellofemoral kinematics. J Orthop Res 2001;19:834-40.

22. Biedert RM, Warnke K. Correlation between the $\mathrm{Q}$ angle and the patella position: a clinical and axial computed tomography evaluation. Arch Orthop Trauma Surg 2001;121:346-9. 
23. Tsakoniti AE, Mandalidis DG, Athanasopoulos SI, Stoupis CA. Effect of Q-angle on patellar positioning and thickness of knee articular cartilages. Surg Radiol Anat 2011;33:97-104.

24. Karvonen RL, Negendank WG, Teitge RA, Reed AH, Miller PR, Fernandez-Madrid F. Factors affecting articular cartilage thickness in osteoarthritis and aging. J Rheumatol 1994;21:1310-8.

25. Huberti HH, Hayes WC. Patellofemoral contact pressures. The influence of q-angle and tendofemoral contact. J Bone Joint Surg Am 1984;66:715-24.

26. Waterton JC, Solloway S, Foster JE, Keen MC, Gandy $\mathrm{S}$, Middleton BJ, et al. Diurnal variation in the femoral articular cartilage of the knee in young adult humans. Magn Reson Med 2000;43:126-32.

27. Hsu RW, Himeno S, Coventry MB, Chao EY. Normal axial alignment of the lower extremity and loadbearing distribution at the knee. Clin Orthop Relat Res 1990;255:215-27.

28. Türkmen F, Acar MA, Kacira BK, Korucu İH, Erkoçak ÖF, Yolcu B, et al. A new diagnostic parameter for patellofemoral pain. Int J Clin Exp Med
2015;8:11563-6.

29. Englund M, Lohmander LS. Patellofemoral osteoarthritis coexistent with tibiofemoral osteoarthritis in a meniscectomy population. Ann Rheum Dis 2005;64:1721-6.

30. Szebenyi B, Hollander AP, Dieppe P, Quilty B, Duddy J, Clarke S, et al. Associations between pain, function, and radiographic features in osteoarthritis of the knee. Arthritis Rheum 2006;54:230-5.

31. Richardson ML, Selby B, Montana MA, Mack LA. Ultrasonography of the knee. Radiol Clin North Am 1988;26:63-75.

32. Spannow AH, Stenboeg E, Pfeiffer-Jensen M, Herlin $\mathrm{T}$. Ultrasound measurement of joint cartilage thickness in large and small joints in healthy children: a clinical pilot study assessing observer variability. Pediatr Rheumatol Online J 2007;5:3.

33. Pradsgaard DØ, Fiirgaard B, Spannow AH, Heuck C, Herlin T. Cartilage thickness of the knee joint in juvenile idiopathic arthritis: comparative assessment by ultrasonography and magnetic resonance imaging. J Rheumatol 2015;42:534-40. 